\title{
Regenerated gratings in air-hole microstructured fibers for high-temperature pressure sensing
}

\author{
Tong Chen, ${ }^{1}$ Rongzhang Chen, ${ }^{1}$ Charles Jewart, ${ }^{1}$ Botao Zhang, ${ }^{1}$ Kevin Cook, ${ }^{2}$ John Canning, ${ }^{2}$ and Kevin P. Chen ${ }^{1, *}$ \\ ${ }^{1}$ Department of Electrical Engineering, University of Pittsburgh, Pittsburgh, Pennsylvania 15261, USA \\ ${ }^{2}$ Interdisciplinary Photonics Laboratories, School of Chemistry, University of Sydney, Sydney NSW 1430, Australia \\ ${ }^{*}$ Corresponding author: kchen@engr.pitt.edu \\ Received June 15, 2011; revised August 10, 2011; accepted August 12, 2011; \\ posted August 15, 2011 (Doc. ID 149303); published September 6, 2011
}

\begin{abstract}
We present thermally regenerated fiber Bragg gratings in air-hole microstructured fibers for high-temperature, hydrostatic pressure measurements. High-temperature stable gratings were regenerated during an $800{ }^{\circ} \mathrm{C}$ annealing process from hydrogen-loaded Type I seed gratings. The wavelength shifts and separation of grating peaks were studied as functions of external hydrostatic pressure from 15 to $2400 \mathrm{psi}$, and temperature from $24^{\circ} \mathrm{C}$ to $800^{\circ} \mathrm{C}$. This Letter demonstrates a multiplexible pressure and temperature sensor technology for high-temperature environments using a single optical fiber feedthrough. (c) 2011 Optical Society of America

OCIS codes: $\quad 060.2370,060.4005,120.5475,120.6780$.
\end{abstract}

Sensors that operate at high temperatures are needed for a wide range of applications in the energy, automobile, and aerospace industries. For example, fast, accurate, and reliable interrogation of gas pressure information ensures safe and efficient operations of gas turbine, coal boilers, and power plants, where the operating temperatures range from $400{ }^{\circ} \mathrm{C}$ to more than $1000^{\circ} \mathrm{C}$. Optical fiber sensors have always been considered good candidates for harsh environment applications. A single Fabry-Perot interferometer implemented on a fiber tip can perform pressure or temperature sensing beyond $1000^{\circ} \mathrm{C}[1]$.

Compared with fiber interferometer sensors, fiber Bragg gratings (FBGs) offer greater multiplexing capability [2], and continuous efforts have been made to improve the operating temperature of FBGs. These may involve modifying the chemical composition of the fiber core [3], and using an ultrafast laser to form a Type II damaged grating [4]. Previously, we reported a high-temperature pressure fiber sensor in which the grating was inscribed in an air-hole microstructure fiber with an ultrafast laser [5]. The Type II FBG shows stable and reproducible pressure sensing operation over $800^{\circ} \mathrm{C}$. But further optimization of grating linewidth and suppression of the strong laser-induced birefringence are needed to allow better pressure sensing range and accuracy. The relatively large in-line loss with Type II permanent damage also limits sensor multiplexing.

Recently, a new type of high-temperature FBG was reported, in which grating structures are regenerated after the Type I seed gratings are erased during a hightemperature annealing process [6-10]. Unlike chemical composition gratings, it has been shown that the regeneration process is independent from dopants in fiber cores [] $]$ and photosensitization processes, such as hydrogen loading [10]. This versatility opens up hightemperature stabilization to other material systems and allows postregeneration of unloaded gratings, including arrays of online draw tower gratings [10]. By carefully controlling the strength of the seed grating and annealing procedures, high-temperature gratings with $\sim 35 \%$ reflectivity and narrow linewidth can be regenerated [7]. Stable operating temperature up to $1295^{\circ} \mathrm{C}$ was reported for regenerative gratings [8]. The ultrahigh temperature stability, good grating qualities, and relatively simple procedures make the regenerated gratings technique attractive to multiplexible high-temperature sensing applications.

In this Letter, we report for the first time, to our knowledge, a regenerated grating in an air-hole microstructured fiber, and present a multiplexible fiber sensor able to accurately measure hydrostatic pressure and high temperature at the same time. The regenerated grating is stable at $800^{\circ} \mathrm{C}$, and shows a sharp linewidth and minimum intrinsic birefringence. Hydrostatic gas pressure from 15-2400 psi (1-165 bars) were measured with an operating temperature range from $24-800^{\circ} \mathrm{C}$. The pressure response is discriminated from temperature response by interrogating both the wavelength shifts and the birefringence separation of the grating peaks. Multiplexing a large number of regenerated grating sensors in a single fiber provides a simple and reliable solution for distributive and simultaneous measurement of a wide range of temperature and pressure in high-temperature harsh environments.

Air-hole fibers used in this work are $220 \mu$ m diameter two-hole fibers with two $90 \mu \mathrm{m}$ air holes, as shown in the upper-right inset of Fig. 1(a). The fiber core is $9.7 \mu \mathrm{m} \times$ $7.5 \mu \mathrm{m}$ in size and $4.5 \mu \mathrm{m}$ away from the geometric center of the fiber. The $\mathrm{GeO}_{2}$ concentration is $\sim 10 \mathrm{~mol}$. \% in the core. A piece of $20 \mathrm{~cm}$ long air-hole fiber was fusion spliced at both ends with conventional single-mode fiber (Corning SMF-28). Multiple resplices were performed to seal the air holes from ambient pressure. The spliced fiber was loaded in a hydrogen chamber at $T \sim 25^{\circ} \mathrm{C}$, $P \sim 2400$ psi (165 bars) for $t \sim 2$ weeks. An $L=3 \mathrm{~cm}$ long FBG with $\lambda_{\text {Bragg }} \sim 1550 \mathrm{~nm}$ was inscribed into the fiber core using a $248 \mathrm{~nm} \mathrm{KrF}$ laser and a phase mask with $\Delta=1060 \mathrm{~nm}$. With a cumulative fluence of $\sim 22,500$ pulses at $\sim 50 \mathrm{~mJ} / \mathrm{cm}^{2}$ radiation intensity, the seed FBG was inscribed into the fiber core with $R \sim 97.5 \%$, as shown in the upper-left inset of Fig. 1(a). The excessive UV exposures induced $\Delta n_{\mathrm{DC}}>10^{-3}$ index change in fiber core.

The seed FBG was then annealed at $T=800{ }^{\circ} \mathrm{C}$. The evolution of the grating spectral shape is shown in Fig. 1(a), and the changes in $\lambda_{\text {Bragg }}$ and $R$ in Fig. 1(b). Five 


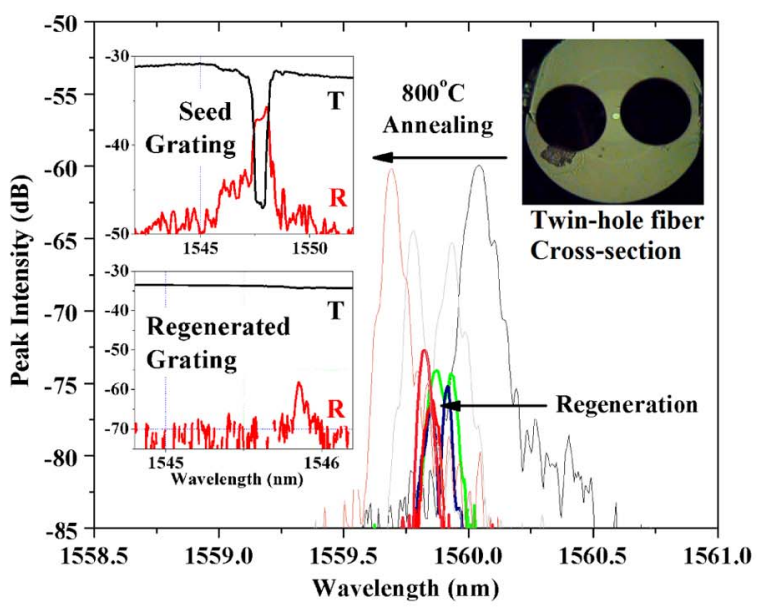

(a)

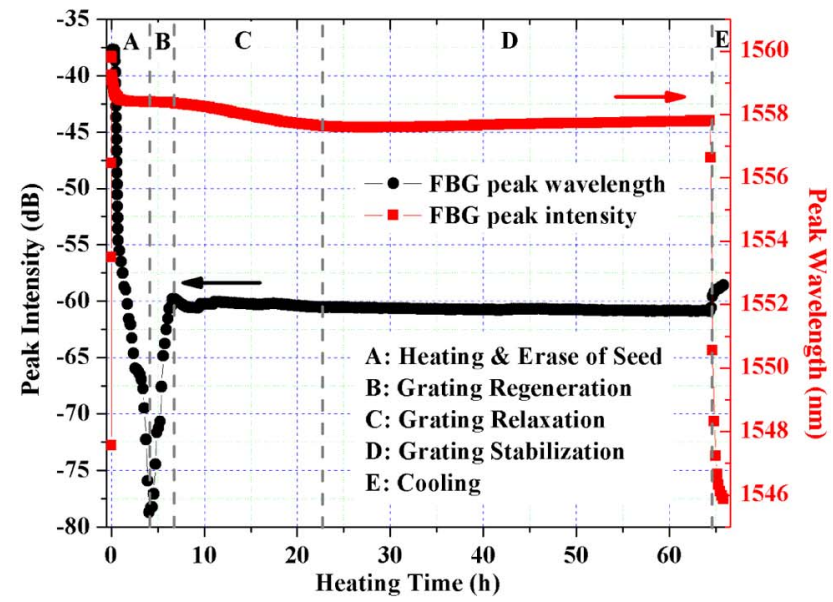

(b)

Fig. 1. (Color online) Evolution of (a) grating reflection spectra and (b) grating strength and resonate wavelength during the regeneration at $800^{\circ} \mathrm{C}$. Insets, microscopic cross-section image of the twin-hole fiber, and transmission and reflection of the seed and regenerated gratings.

steps were identified in the regeneration process. The erasure of the seed FBG in the first $4.5 \mathrm{~h}$ was labeled as step $\mathrm{A}$, followed by the grating regeneration in the next $2 \mathrm{~h}$, as is labeled step B. During regeneration, a blueshift in $\lambda_{\text {Bragg }}$ was observed, which was attributed to relaxation of UV-induced $\Delta n_{\mathrm{DC}}$. The regenerated grating continued relaxing in the next $15 \mathrm{~h}$, labeled step $\mathrm{C}$, which was followed by $40 \mathrm{~h}$ of grating stabilization in step D. After cooling down in step $\mathrm{E}$, a regenerated grating with $R \sim 0.23 \%$ was obtained at $24^{\circ} \mathrm{C}$, as is shown in the lower-left inset of Fig. 1(a). The index modulation ratio, $\Delta n_{\text {reg }} / \Delta n_{\text {seed }} \sim 1.9 \%$, between regenerated and seed grating is lower than that reported for regeneration within standard fibers where $\Delta n_{\text {reg }} / \Delta n_{\text {seed }} \sim 10 \%$ [ $\left.\underline{7}-\underline{9}\right]$. This is probably due to several factors, including optical mode overlap with the core/cladding [10], as well as the far more rapid annealing we used.

Several heating and cooling cycles were carried out over $\left(24^{\circ} \mathrm{C}-800^{\circ} \mathrm{C}\right)$ to test the thermal stability of the regenerated grating, and no hysteresis was observed. In a temperature range from $400^{\circ} \mathrm{C}-800^{\circ} \mathrm{C}, \lambda_{\text {Bragg }}$ shifted linearly as a function of temperature where $\partial \lambda / \partial T \sim$ $0.01518 \mathrm{~nm} /{ }^{\circ} \mathrm{C}$, as is shown in Fig. 2 .

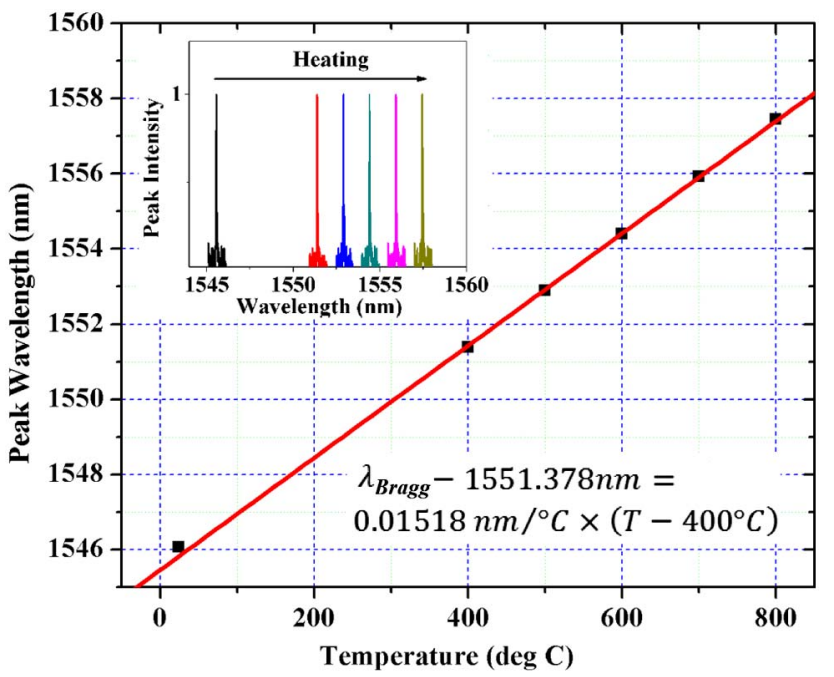

Fig. 2. (Color online) Wavelength shift of the regenerated grating with respect to the change of temperature. Inset, grating reflection spectra at $24,400,500,600,700$, and $800{ }^{\circ} \mathrm{C}$.

The pressure response of the regenerated grating was measured at $800^{\circ} \mathrm{C}$, as shown in Fig. 3. Under room pressure of $P=15$ psi (1 bar), a sharp grating with FWHM linewidth $\Delta \lambda<40 \mathrm{pm}$ in the reflection spectrum is observed. Using an optical spectrum analyzer (Ando AQ6317B with $20 \mathrm{pm}$ resolution) and an in-line U-bench polarization analyzer, the FBG peak split under room pressure cannot be resolved, which suggests a small intrinsic birefringence $\left(<10^{-5}\right)$ induced by the laser writing and the regenerative process. This is a 30 -fold improvement over high-temperature FBGs inscribed by the ultrafast laser in the same fiber [5]. When a hydrostatic pressure is applied to the twin-hole fiber using $\mathrm{N}_{2}$ gas, the deformation of the air holes transfers the external pressure into internal stress birefringence in the fiber core, and the grating peak split into orthogonal polarizations. $\Delta \lambda_{\text {Bragg }}=180 \mathrm{pm}$ was obtained under $P=$ $2400 \mathrm{psi}$ (165 bars) at $T=800^{\circ} \mathrm{C}$, which corresponds to a birefringence change $\Delta B \sim 1.2 \times 10^{-4}$. The $\lambda_{\text {Bragg }}$ shifts linearly for both polarization eigenmodes (TE and TM) with applied external pressure over the entire testing range at $800^{\circ} \mathrm{C}$, as is shown in Fig. $\underline{4(\mathrm{a})}$. Figure $\underline{4(\mathrm{~b})}$

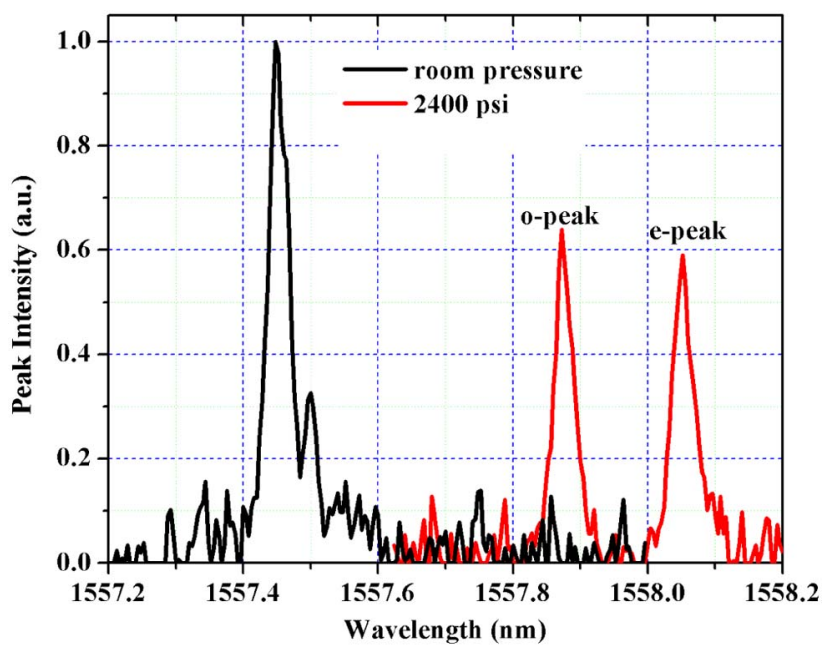

Fig. 3. (Color online) Pressure response of the regenerated grating at $800^{\circ} \mathrm{C}$ : black, room pressure of $15 \mathrm{psi}$; red, $2400 \mathrm{psi}$. 


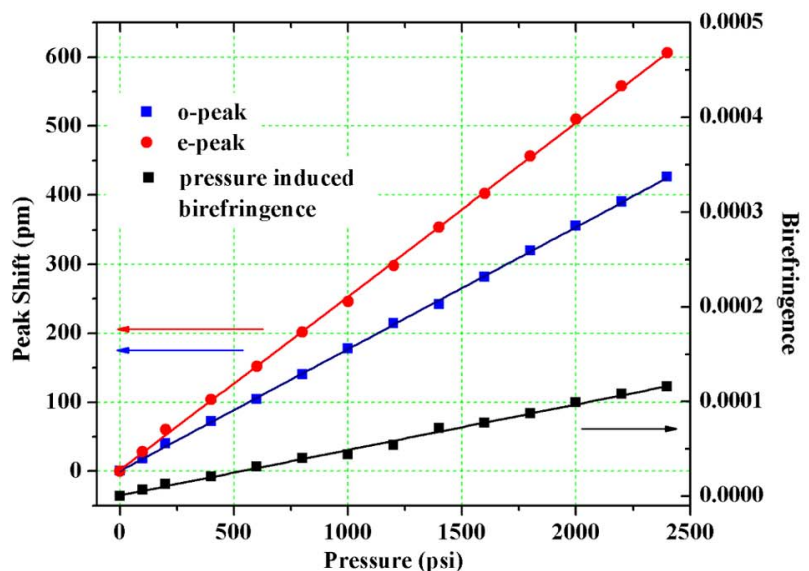

(a)

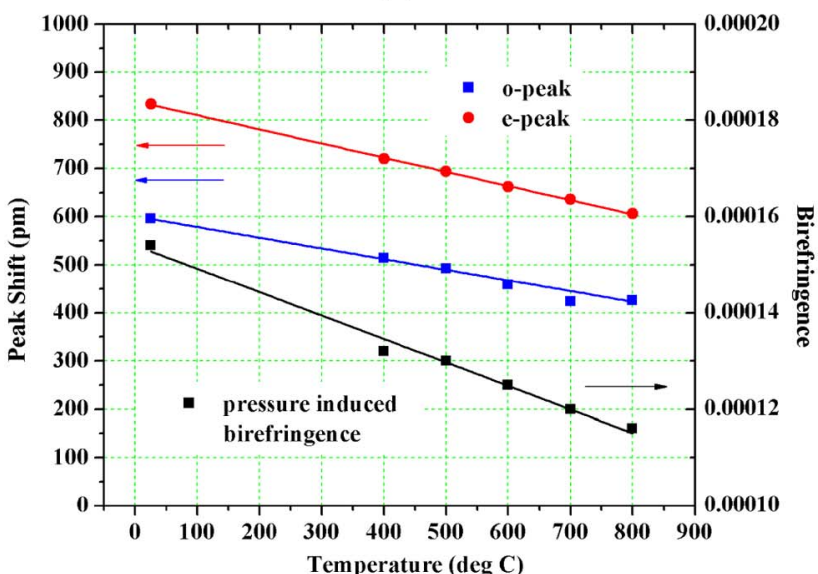

(b)

Fig. 4. (Color online) Sensor responses versus (a) pressure from 15 to $2400 \mathrm{psi}$ at $800{ }^{\circ} \mathrm{C}$; (b) temperature from $24^{\circ} \mathrm{C}$ to $800{ }^{\circ} \mathrm{C}$ under pressure of 2400 psi.

shows the pressure response, where $P=2400$ psi (165 bars) at different temperatures. The pressure sensitivity of the grating is $\partial \lambda / \partial T=13.3 \mathrm{psi} / \mathrm{pm}$ at $T=800^{\circ} \mathrm{C}$, which is $\sim 25 \%$ lower than the sensitivity at room temperature, $T=24{ }^{\circ} \mathrm{C}$. This is explained as being due to the high-temperature softening of the glass fiber structure and a decrease of strain-optic coefficients.

Using FBG peak shifts and pressure-induced peak separations, temperature and pressure can be measured simultaneously. The sensor response matrix is expressed as

$$
\begin{aligned}
\left(\Delta \lambda_{o, e}\right)= & \left(\partial \lambda_{o, e} / \partial T\right) \Delta T \\
& +\left(\partial \lambda_{o, e} / \partial P+\left(\partial^{2} \lambda_{o, e} / \partial T \partial P\right) \Delta T\right) \Delta P \\
\partial \lambda_{o, e} / \partial T= & 1.518 \times 10^{-4}\left(\mathrm{~nm} /{ }^{\circ} \mathrm{C}\right) \\
\left(\begin{array}{l}
\partial \lambda_{o} / \partial P \\
\partial \lambda_{e} / \partial P
\end{array}\right)= & \left(\begin{array}{l}
2.521 \times 10^{-4} \\
3.526 \times 10^{-4}
\end{array}\right)(\mathrm{nm} / \mathrm{psi}) \\
\left(\begin{array}{l}
\partial^{2} \lambda_{o} / \partial T \partial P \\
\partial^{2} \lambda_{e} / \partial T \partial P
\end{array}\right)= & \left(\begin{array}{l}
-9.185 \times 10^{-8} \\
-1.232 \times 10^{-7}
\end{array}\right)\left(\mathrm{nm} / \mathrm{psi} /{ }^{\circ} \mathrm{C}\right)
\end{aligned}
$$

in which the temperature response coefficient $\partial \lambda / \partial T$, pressure response coefficients $\partial \lambda / \partial P$, and the temperature-dependent pressure response coefficients $\partial^{2} \lambda / \partial T \partial P$ are calibrated at $\lambda_{\text {Bragg }}=1551.378 \mathrm{~nm}$ with $T=400{ }^{\circ} \mathrm{C}$ and $P=15$ psi ( 1 bar).

A narrow linewidth grating $(<40$ pm FWHM) with minimal intrinsic birefringence $\left(<2 \times 10^{-5}\right)$ enables highresolution pressure and temperature measurements. With a tunable laser of $0.1 \mathrm{pm}$ resolution, it is possible to obtain $\Delta T \sim 0.01{ }^{\circ} \mathrm{C}$ temperature resolution and $\Delta P \sim$ $1.5 \mathrm{psi}$ (0.1 bar) pressure resolution simultaneously. This is a significant performance enhancement compared with Type II grating results [5]. The low in-line loss and ultrasharp linewidth of the regenerated grating also provide wide flexibility in sensor multiplexing. The maximum temperature and pressure in our test were limited by the apparatus. It is believed that air-hole silica fiber has potential to sustain much higher pressure, up to 10,000 psi (690 bars), according to our simulation. With improvement in seed grating strength and optimization of the annealing schedule, it is possible to push the grating operating temperature to $T>1000^{\circ} \mathrm{C}[\underline{7}, \underline{8}]$.

In summary, this Letter applies the thermal regeneration technique to produce high-temperature stable FBG pressure and temperature sensors for industry applications using a well-known pressure sensor designs imbedded in twin-hole fiber. The sensor operates for a pressure range of $15-2400$ psi (1-165 bars) and a temperature range of $24^{\circ} \mathrm{C}-800^{\circ} \mathrm{C}$. In comparison with other high-temperature pressure sensors, the regenerated gratings in air-hole fibers are accurate, reliable, and can be easily multiplexed. A pressure and temperature sensing network can be formed with large numbers of these sensors on a single fiber feedthrough, and interrogated with a single optical sensing unit.

This work was supported by the National Science Foundation (NSF, CMMI-0644681 and CMMI-0900564) and the Department of Energy (DOE, DE-FE0003859)

\section{References}

1. J. Yi, E. Lally, A. Wang, and Y. Xu, IEEE Photon. Technol. Lett. 23, 9 (2011).

2. K. O. Hill and G. Meltz, J. Lightwave Technol. 15, 1263 (1997).

3. M. Fokine, J. Opt. Soc. Am. B 19, 1759 (2002).

4. D. Grobnic, C. W. Smelser, S. J. Miharlov, and R. B. Walker, Meas. Sci. Technol. 17, 1009 (2006).

5. C. M. Jewart, Q. Wang, J. Canning, D. Grobnic, S. J. Mihailov, and K. P. Chen, Opt. Lett. 35, 1443 (2010).

6. B. Zhang and M. Kahrizi, IEEE Sens. J. 7, 586 (2007).

7. S. Bandyopadhyay, J. Canning, M. Stevenson, and K. Cook, Opt. Lett. 33, 1917 (2008).

8. S. Bandyopadhyay, J. Canning, P. Biswas, M. Stevenson, and K. Dasgupta, Opt. Express 19, 1198 (2011).

9. J. Canning, M. Stevenson, S. Bandyopadhyay, and K. Cook, Sensors 8, 6448 (2008).

10. E. Lindner, J. Canning, C. Chojetzki, S. Brückner, M. Becker, M. Rothhardt, and H. Bartelt, Appl. Opt. 50, 2519 (2011). 